\title{
Antenatal Betamethasone Administration Decreases the Lung Hyaluronan Concentration in Preterm Rabbit Pups
}

\author{
HANS JOHNSSON, LARS ERIKSSON, AND GUNNAR SEDIN \\ Departments of Women's and Children's Health [H.J., G.S.], Medical Biochemistry and Microbiology \\ [H.J.], Physiology [H.J., G.S.], and Genetics and Pathology [L.E.], Uppsala University, SE-751 85 \\ Uppsala, Sweden.
}

\begin{abstract}
ABST
Maternal treatment with corticosteroids before preterm deliv-
ery is effective in reducing the incidence of respiratory distress
syndrome and neonatal mortality. We hypothesized that cortico-
steroids might lower the lung hyaluronan concentration. Twenty-
five rabbit dams (term $=31 \mathrm{~d}$ ) with timed pregnancies were
injected s.c. with $0.75 \mathrm{mg}$ of betamethasone or saline (controls)
$1 \mathrm{~d}$ before delivery. In addition, two dams delivered at $25 \mathrm{~d}$ of
gestation were injected with 0.75 mg of betamethasone on two
consecutive days before delivery. A total of 238 live pups were
delivered by preterm cesarean section at $25,27,28$, or $29 \mathrm{~d}$ of
gestation and killed immediately. Their lung hyaluronan concen-
trations were measured with a radiometric assay, and wet/dry
lung weight ratios were determined. Lungs of rabbit pups ex-
posed antenatally to betamethasone and delivered at 25 or $27 \mathrm{~d}$ of
\end{abstract}
Antenatal corticosteroid administration to the mother before preterm birth is associated with a reduced incidence of respiratory distress syndrome and a lower neonatal mortality (1-5). This is generally considered to be caused by a steroid-induced increase in surfactant synthesis or release and accelerated morphologic development (6). Accelerated lung cell maturation after steroid exposure has been observed in histologic sections of lungs from rabbits (7). In explant cultures of human fetal lung tissue, increased differentiation (8) and an increase in the capacity of the antioxidant system of the lung to withstand hyperoxia (9) have been noted after exposure to steroids. Antenatally administered corticosteroids also increase the distensibility of the airways (10), an effect at least partially related to the thinning out of the pulmonary interstitium (11). The effects of antenatally administered corticosteroids vary considerably depending on the species, GA at exposure, and steroid

Received June 20, 2000; accepted October 18, 2000.

Correspondence and reprint requests: Gunnar Sedin, M.D., Ph.D., Perinatal Research Laboratory, Uppsala University Children's Hospital, SE-751 85 Uppsala, Sweden; e-mail: gunnar.sedin@kbh.uu.se

Supported by the Swedish Medical Research Council (grant 04998) and HKH Kronprinsessans Lovisas förening för barnsjukvård (HRH Crown Princess Lovisa's Association for Child Health Care). gestation, but not at 28 or $29 \mathrm{~d}$, displayed significantly ( $p=$ 0.001 and $p=0.008$, respectively) lower hyaluronan concentrations than control pups, accompanied by less intense subepithelial staining for hyaluronan in alveolar walls. There was no significant difference in wet/dry lung weight ratio between pups exposed to one dose of betamethasone and controls. Antenatal corticosteroid exposure lowers the lung hyaluronan concentration in preterm rabbit pups delivered at 25 or $27 \mathrm{~d}$ of gestation, but not in those delivered at 28 or 29 d. (Pediatr Res 49: 566-571, 2001)

\section{GA, gestational age \\ W/D, wet/dry lung weight ratio}

Abbreviations:

dosage (12). The effects of antenatal glucocorticoid administration on fetal lung morphology and function have been most extensively studied in the rabbit and sheep. In preterm rabbits, improved postnatal lung function after maternal corticosteroid administration has been observed even without an increase in the surfactant content of lung tissue or alveoli (13-15).

In the lung there are connective tissue fibers in the walls of the pleura, conducting airways, blood vessels, and lymphatics and in the lobular septa, and minute amounts are found in the alveolar walls. Elastic and collagen fibers are equally prominent in most lung connective tissue, except in the alveoli, in which the former predominate. A relative increase in lung interstitial tissue takes place up to the terminal stages of gestation (16). Hyaluronan is a major component of the extracellular matrix, with many important functions. Hyaluronan is a large glycosaminoglycan polymer of hyalobiuronic acid, a disaccharide consisting of glucuronic acid and $N$-acetylglucosamine, with a molecular weight in tissues of 1-10 MD. It forms long unbranched chains with a random coil structure and a high capacity to immobilize water. The concentration of hyaluronan in different tissues varies widely. It appears in abundance in tissues during fetal development or repair, and 
during inflammatory processes (17). The lung hyaluronan concentration in rabbit fetuses normally decreases progressively toward term (18), but may increase in association with neonatal pulmonary diseases, including respiratory distress syndrome (19), and after hyperoxia (20).

We hypothesized that the effects of antenatal corticosteroid administration to the dam on the fetal rabbit lung include a reduction of the lung hyaluronan concentration. The aim of this study was to determine whether antenatal exposure to a corticosteroid lowers the lung hyaluronan concentration and alters the hyaluronan distribution within the lung at birth in preterm rabbit pups. Betamethasone was chosen, as it is the agent recommended for the prevention of respiratory distress syndrome in the human infant. The dose, calculated as milligrams per kilogram of maternal weight (see Methods), was comparable to that used in humans. The dose was not adjusted according to the individual maternal weight, as this is not current clinical practice (21). We chose the rabbit in this study because we have had previous experience with a similar model, used for determining the effect of oxygen exposure on lung hyaluronan (20). Two preliminary reports of the current study have been presented $(22,23)$.

\section{METHODS}

New Zealand White rabbits (normal gestation period, $31 \mathrm{~d}$ ) were studied. Female rabbits (weight, approximately $3 \mathrm{~kg}$ ) were allowed to mate for a few hours, and this was considered d 0 of gestation. Twenty-five dams were injected s.c. at 24,26 , 27 , or $28 \mathrm{~d}$ of gestation with a single dose of betamethasone $0.75 \mathrm{mg}$ (Betapred $^{\mathrm{TM}}$, Glaxo Wellcome, Mölndal, Sweden) in $1 \mathrm{~mL}$ sodium chloride $9 \mathrm{~g} / \mathrm{L}(154 \mathrm{mM})$, or with sodium chloride alone (controls). The relative weight of pups per dam in the case of nine pups of $20 \mathrm{~g}$ (total wt, $180 \mathrm{~g}$ ) and a dam of $3 \mathrm{~kg}$, equals that of a $3-\mathrm{kg}$ baby and a $50-\mathrm{kg}$ mother $(0.06)$. Pups were delivered by cesarean section on the day after the injection. The mean length of time from the injection of betamethasone or sodium chloride to delivery was $28.0 \mathrm{~h}(n=$ $127)$ and $27.3 \mathrm{~h}(n=105)$, respectively. In addition, two rabbit dams were injected with a $0.75-\mathrm{mg}$ dose of betamethasone on each of two consecutive days, at 23 and $24 \mathrm{~d}$ of gestation, and were subsequently delivered by cesarean section on d 25 of gestation.

A total of 27 dams were injected, and 238 live pups were delivered. The numbers of dams and pups and the mean numbers of pups per dam delivered at different GAs and exposed to one or two doses of corticosteroid or to saline were as follows:

GA 25 d - two doses of betamethasone: two dams, 22 pups, $11.0 \mathrm{pups} / \mathrm{dam}$; one dose of betamethasone: three dams, 26 pups, 8.7 pups/dam; saline: three dams, 26 pups, 8.7 pups/dam.

GA $27 \mathrm{~d}$ - one dose of betamethasone: four dams, 29 pups, 7.3 pups/dam; saline: three dams, 23 pups, 7.7 pups/dam.

GA $28 \mathrm{~d}$-one dose of betamethasone: three dams, 28 pups,

9.3 pups/dam; saline: three dams, 27 pups, 9.0 pups/dam.

GA $29 \mathrm{~d}$-one dose of betamethasone: three dams, 29 pups,

9.7 pups/dam; saline: three dams, 28 pups, 9.3 pups/dam.
In addition, five pups were stillborn, two whose mothers had received one dose of betamethasone and three whose mothers had received saline. The sex of the pups was not recorded.

For the cesarean section, the dam was given $1-2 \mathrm{~mL}$ of ketamine $50 \mathrm{mg} / \mathrm{mL}$ (Ketalar ${ }^{\circledR}$, Parke-Davies, Barcelona, Spain) through an ear vein, followed by spinal or epidural anesthesia produced by $1.5-2.0 \mathrm{~mL}$ of bupivacaine $5 \mathrm{mg} / \mathrm{mL}$ (Marcain ${ }^{\circledR}$ spinal, Astra, Södertälje, Sweden). Pups were killed immediately after birth by an injection of $0.5-1 \mathrm{~mL}$ of thiopental sodium $50 \mathrm{mg} / \mathrm{mL}$ (Pentothal ${ }^{\circledR}$ Natrium, Abbott, North Chicago, IL, U.S.A.) given s.c. in the nuchal region, and a string was subsequently tied around the neck to prevent fluid escape from the airways. The pup weight was recorded, and the lungs were then removed en bloc. No ties were placed around the trachea or bronchi when the lungs were removed.

The right lung was fixed in a formalin buffer with microwave heating, according to the method described by Hellström et al. (24). In each group, from the five lungs that had a concentration of hyaluronan/dry lung weight closest to the mean concentration in that group, 5- to 6- $\mu$ m-thick cryostat sections were taken and stained with hematoxylin and eosin, and labeled in situ for hyaluronan with a biotinylated hyaluronan-binding protein according to the method of Ripellino et al. (25) as described earlier (18). Briefly, specificity of the staining for hyaluronan was monitored by two types of controls: 1) Rat kidney sections were processed along with each series of rabbit lung section. Hyaluronan forms a characteristic staining pattern in the rat kidney, and demonstration of this typical pattern was required to consider the preparation of the series acceptable. 2) One section of lung tissue incubated with Streptomyces hyaluronidase (Seikagaku Kogyo, Tokyo, Japan), $500 \mathrm{U} / \mathrm{mL}$ for $2 \mathrm{~h}$ at $37^{\circ} \mathrm{C}$ before staining, was processed in parallel with two adjacent sections of each lung sample, together with a rat kidney section. In acceptable series the hyaluronidase-treated sections did not exhibit any staining by the biotinylated hyaluronan-binding protein. The intensity of the hyaluronan staining of the visceral pleura and of the arterioles, bronchioli, and alveolar interstitial space was semiquantified on a 0 to 3 scale as described previously (18). This was performed by one of the authors (L.E.), who was not informed about the background data in each section.

The left lung was weighed and frozen, and then freeze-dried for $72 \mathrm{~h}$. The dried lung was reweighed, and the water content was calculated as the W/D ratio. The dried lungs were then digested with Pronase (protease P-5147, Streptomyces griseus, Sigma Chemical Co., St. Louis, MO, U.S.A.) in a $2.5 \mathrm{U} / \mathrm{mL}$ buffer solution [0.05 M Tris (hydroxymethyl) aminomethane, $0.01 \mathrm{M} \mathrm{CaCl}_{2}, \mathrm{pH} 7.2$ ] at $55^{\circ} \mathrm{C}$ overnight $(>16 \mathrm{~h})$. One unit of enzyme was added for each $10 \mathrm{mg}$ of dry lung material. The digestion was ended by heating to $100^{\circ} \mathrm{C}$ for $10 \mathrm{~min}$ in a waterbath. The hyaluronan content was determined with a radiometric assay kit (HA 50, Pharmacia, Uppsala, Sweden) (26), and the hyaluronan concentration was calculated in relation to the dry and wet lung weights.

Data are presented as mean \pm SEM. Statistical analysis of continuous variables was performed with one-way ANOVA, followed by either the Bonferroni (equal variance) or the Tamhane T2 (unequal variances) post hoc test. For all statis- 
tical analyses the software SPSS version 8.0 (SPSS, Chicago, IL, U.S.A.) was used. In all tests the level of statistical significance was set at $p<0.05$.

This study was approved by the Uppsala University Animal Trials Ethics Committee (project no. C 226/96).

\section{RESULTS}

Lung hyaluronan concentration. The hyaluronan concentrations in the lungs, calculated as micrograms of hyaluronan per gram of dry lung weight, are given in Table 1. The effects of exposure to one dose of betamethasone are also shown in Figure 1.

Pups delivered at GA $25 \mathrm{~d}$ of dams injected antenatally with one dose of betamethasone $(n=26)$ showed a significantly lower $(p=0.001)$ mean hyaluronan concentration (531.5 \pm $32.9 \mu \mathrm{g} / \mathrm{g}$ dry lung wt) than the control pups $(763.0 \pm 46.3$ $\mu \mathrm{g} / \mathrm{g}$ dry lung wt; $n=26$ ). Pups of dams injected antenatally with two doses of betamethasone $(n=22)$ also had a significantly lower $(p=0.022)$ mean hyaluronan concentration $(595.2 \pm 28.9 \mu \mathrm{g} / \mathrm{g}$ dry lung wt) than the controls. The difference in lung hyaluronan between the group exposed to one dose of corticosteroid and the group exposed to two doses was not significant ( $p=0.629$; Table 1$)$.

Pups delivered at GA $27 \mathrm{~d}$ of dams injected antenatally with betamethasone $(n=29)$ showed a significantly lower $(p=$ 0.008 ) hyaluronan concentration (382.4 $\pm 17.3 \mu \mathrm{g} / \mathrm{g}$ dry lung wt) than the control pups (494.2 $\pm 25.4 \mu \mathrm{g} / \mathrm{g}$ dry lung wt; $n=$ 23; Table 1).

Pups delivered at GA 28 or $29 \mathrm{~d}$ of dams injected antenatally with betamethasone had a mean lung hyaluronan concentration that was not different from that in the control pups of the same GA (Table 1).

Lung hyaluronan staining. Scoring of the hyaluronan staining $(0-3)$ showed gradually weaker staining in the alveolar walls with increasing maturity. In the pleura there was virtually no staining for hyaluronan in any group (Table 2). Among pups delivered at GA $25 \mathrm{~d}$, the staining for hyaluronan in the alveolar walls was much less intense in those exposed to betamethasone antenatally than in controls of the same maturity. Photomicrographs of sections from lungs of rabbit pups delivered at GA 25 d, stained with biotinylated hyaluronanbinding protein, are presented in Figure 2. There was intense subepithelial staining for hyaluronan in the alveolar walls in

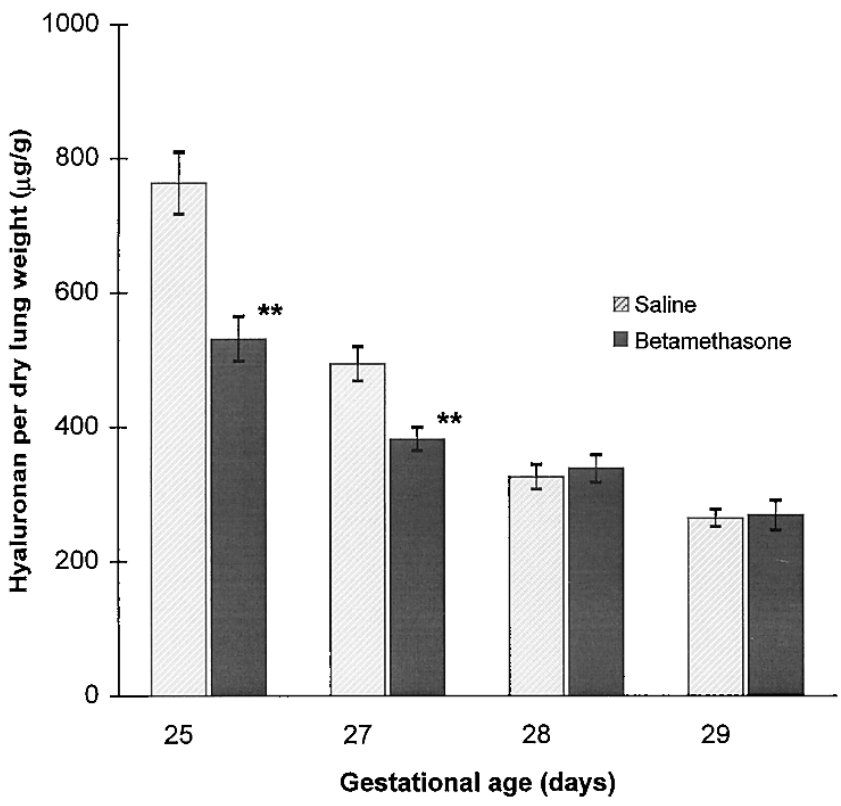

Fig. 1. Histogram of the mean ( \pm SEM) lung hyaluronan concentration/dry lung weight $(\mu \mathrm{g} / \mathrm{g})$ at birth in preterm rabbit pups exposed to one dose of betamethasone or saline (controls), administered to the dam on the day preceding delivery $(* * p<0.01$, compared with controls of the same GA).

control pups, but this staining was much less intense in the pups exposed to betamethasone. The same was true for the arterial walls in the lungs.

Among pups born at GA $27 \mathrm{~d}$, the staining for hyaluronan in the bronchioli was more distinct in control pups than in pups exposed to betamethasone. The corresponding staining in the arterial and alveolar walls differed less markedly between these two groups of pups.

In pups born at GA 28 or 29 d, no obvious difference in staining for hyaluronan was observed in the above-mentioned structures between control pups and pups exposed to betamethasone.

Lung water. The lung W/D ratio $(9.0 \pm 0.1)$ in GA $25-\mathrm{d}$ pups of dams injected antenatally with two doses of betamethasone was significantly higher $(p=0.001)$ than the ratio $(8.4 \pm$ $0.1)$ in the controls, and was also significantly higher $(p=$ $0.002)$ than the ratio $(8.5 \pm 0.1)$ in pups of dams that received one dose of betamethasone. There were no other significant differences in lung W/D ratio between groups exposed to

Table 1. Body and lung weights and lung hyaluronan concentrations

\begin{tabular}{|c|c|c|c|c|c|c|c|c|}
\hline $\begin{array}{l}\text { GA } \\
\text { (d) }\end{array}$ & $\begin{array}{l}\text { Antenatal } \\
\text { injection }\end{array}$ & $n$ & Body wt (g) & Wet lung wt (g) & Dry lung wt (g) & $\begin{array}{l}\text { Wet/Dry } \\
\text { lung wt }\end{array}$ & $\begin{array}{c}\text { HA/wet lung } \\
\text { wt }(\mu \mathrm{g} / \mathrm{g})\end{array}$ & $\begin{array}{l}\text { HA/dry lung } \\
\text { wt }(\mu \mathrm{g} / \mathrm{g})\end{array}$ \\
\hline 25 & saline & 26 & $18.4 \pm 0.5$ & $0.238 \pm 0.009$ & $0.029 \pm 0.001$ & $8.4 \pm 0.1$ & $91.6 \pm 5.8$ & $763.0 \pm 46.3$ \\
\hline 25 & betameth. & 26 & $16.2 \pm 0.5^{*}$ & $0.211 \pm 0.011$ & $0.025 \pm 0.001$ & $8.5 \pm 0.1$ & $62.9 \pm 4.2 \dagger$ & $531.5 \pm 32.9 \dagger$ \\
\hline 25 & betameth. $\times 2$ & 22 & $15.1 \pm 0.4 \div$ & $0.210 \pm 0.010$ & $0.023 \pm 0.001 *$ & $9.0 \pm 0.1 \dagger$ & $66.9 \pm 3.7 \dagger$ & $595.2 \pm 28.9^{*}$ \\
\hline 27 & saline & 23 & $28.6 \pm 0.9$ & $0.357 \pm 0.014$ & $0.039 \pm 0.002$ & $9.1 \pm 0.1$ & $54.5 \pm 2.9$ & $494.2 \pm 25.4$ \\
\hline 27 & betameth. & 29 & $28.6 \pm 1.0$ & $0.364 \pm 0.014$ & $0.040 \pm 0.002$ & $9.3 \pm 0.1$ & $41.4 \pm 2.0 \dagger$ & $382.4 \pm 17.3 \dagger$ \\
\hline 28 & saline & 27 & $32.7 \pm 1.3$ & $0.427 \pm 0.019$ & $0.044 \pm 0.002$ & $9.8 \pm 0.1$ & $33.6 \pm 2.0$ & $326.3 \pm 18.2$ \\
\hline 28 & betameth. & 28 & $25.3 \pm 1.1 \div$ & $0.318 \pm 0.017 \pm$ & $0.034 \pm 0.002 \dagger$ & $9.4 \pm 0.1$ & $36.1 \pm 2.2$ & $338.7 \pm 20.2$ \\
\hline 29 & saline & 28 & $42.7 \pm 1.4$ & $0.507 \pm 0.021$ & $0.054 \pm 0.002$ & $9.4 \pm 0.1$ & $28.4 \pm 1.4$ & $265.2 \pm 12.7$ \\
\hline 29 & betameth. & 29 & $37.2 \pm 1.0^{*}$ & $0.400 \pm 0.018 \dagger$ & $0.046 \pm 0.002 *$ & $8.8 \pm 0.2^{*}$ & $31.5 \pm 2.8$ & $269.7 \pm 22.1$ \\
\hline
\end{tabular}

All values are mean \pm SEM.

Abbreviations: betameth., betamethasone HA, hyaluronan.

$* p<0.05 ; \dagger p<0.01 \ddagger p<0.001$; all compared with controls of the same GA. 
Table 2. Lung hyaluronan staining scores

\begin{tabular}{|c|c|c|c|c|c|c|}
\hline $\begin{array}{l}\text { Gestational } \\
\text { age (d) }\end{array}$ & Antenatal injection & $n$ & Pleura & Arterioli & Bronchioli & Alveoli \\
\hline 25 & saline & 26 & $0.0 \pm 0.0$ & $2.6 \pm 0.2$ & $1.8 \pm 0.2$ & $1.6 \pm 0.2$ \\
\hline 25 & betamethasone & 26 & $0.0 \pm 0.0$ & $2.0 \pm 0.0$ & $1.6 \pm 0.2$ & $0.6 \pm 0.2$ \\
\hline 27 & saline & 23 & $0.5 \pm 0.3$ & $1.4 \pm 0.2$ & $1.4 \pm 0.2$ & $0.6 \pm 0.2$ \\
\hline 27 & betamethasone & 29 & $0.0 \pm 0.0$ & $1.2 \pm 0.4$ & $0.4 \pm 0.2$ & $0.4 \pm 0.2$ \\
\hline 28 & saline & 27 & $0.0 \pm 0.0$ & $1.8 \pm 0.2$ & $1.6 \pm 0.2$ & $0.0 \pm 0.0$ \\
\hline 29 & betamethasone & 29 & $0.0 \pm 0.0$ & $1.4 \pm 0.2$ & $0.6 \pm 0.2$ & $0.0 \pm 0.0$ \\
\hline
\end{tabular}

All values are mean \pm SEM of five pups.
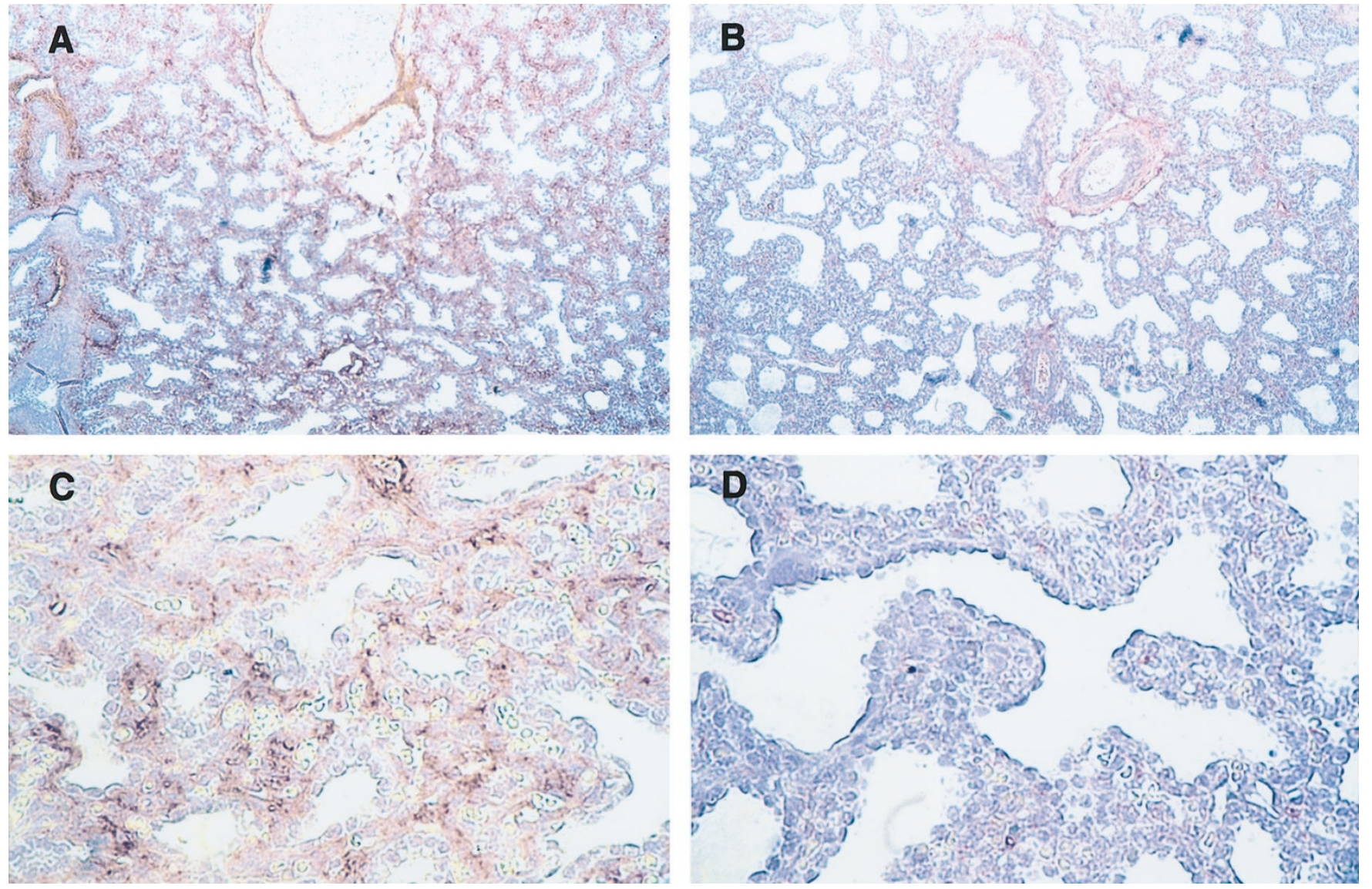

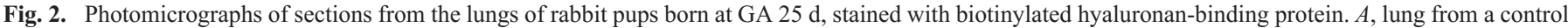

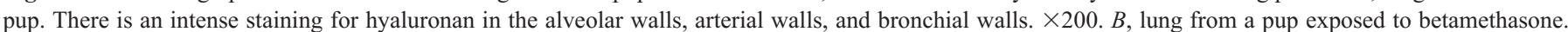

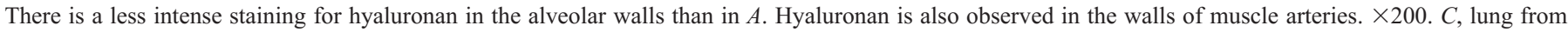

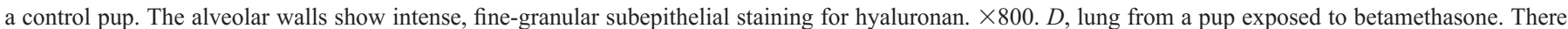
is only weak staining for hyaluronan in the alveolar walls. $\times 800$.

betamethasone antenatally and their respective controls at any of the investigated GAs (Table 1).

Birth weights and lung weights. Among pups delivered at GA 25,28 , or $29 \mathrm{~d}$, pups of dams injected antenatally with one dose of betamethasone had a significantly lower birth weight than controls delivered at the same GA. Among pups delivered at GA $27 \mathrm{~d}$, there was no significant difference in mean birth weight between pups of dams injected antenatally with one dose of betamethasone and control pups. There was no significant difference in birth weight between the groups of pups exposed to one and two doses of betamethasone and delivered at GA 25 d.
Among pups delivered at GA 25 or $27 \mathrm{~d}$, the mean wet lung weight did not differ significantly between the groups. Pups exposed to two doses of betamethasone and delivered at GA $25 \mathrm{~d}$ had a significantly $(p=0.010)$ lower mean dry lung weight than the control pups. There was no significant difference in mean dry lung weight between pups exposed to one dose of betamethasone and their respective controls.

Among pups delivered at GA 28 or $29 \mathrm{~d}$, the mean wet lung weights were significantly lower in pups exposed antenatally to one dose of betamethasone than in control pups of the same GA ( $p<0.001$ and $p=0.002$, respectively). The mean dry lung weights in pups exposed antenatally to betamethasone 
were also significantly lower $(p=0.003$ and $p=0.038)$ than those in the respective controls (Table 1). Calculations using lung weight/body weight instead of just lung weight yielded similar results (data not shown).

\section{DISCUSSION}

The main finding in this study was that preterm rabbit pups exposed to betamethasone and delivered at GA 25 or $27 \mathrm{~d}$ had lower lung hyaluronan concentrations than control pups of corresponding GAs. Among pups delivered at GA 28 or 29 d, there was no difference in the lung hyaluronan concentration between pups exposed antenatally to betamethasone and control pups of corresponding GAs.

The lower lung hyaluronan concentrations found in this study in rabbit pups delivered at GA 25 or $27 \mathrm{~d}$ after antenatal betamethasone administration to the dam could be a consequence of an inhibitory effect of betamethasone on hyaluronan synthesis, or of a direct stimulation of hyaluronan degradation, or both. Either or both of these two effects could in turn constitute one part of the general acceleration of lung maturation that can be induced by antenatal administration of a corticosteroid $(4,6)$. The lack of effect on pups delivered at GA 28 and $29 \mathrm{~d}$ might be explained by a hypothetical GA-related time window of corticosteroid sensitivity (14), or by the almost complete lung maturation at $28 \mathrm{~d}$ of gestation. After direct corticosteroid administration to fetal rabbits 2 or $3 \mathrm{~d}$ before delivery at GA 21-28 d, Kikkawa et al. (7) found that the pulmonary maturation in each GA group was accelerated by 1-1.5 d, up to GA $28 \mathrm{~d}$, when the maturation was almost complete as assessed by microscopic examination. Furthermore, in a study of the effects of maternal administration of betamethasone, Rider et al. (14) noted that the maturational effect on the lung structure was maximal even at the lowest dose of betamethasone used $(0.05 \mathrm{mg} / \mathrm{kg}$, given daily for $2 \mathrm{~d}$ before delivery). These findings could possibly explain why no additional effect was observed in the present study in pups delivered at GA $25 \mathrm{~d}$ whose mothers were given two doses of betamethasone, compared with the effect in corresponding pups exposed to only one dose. In humans, the effect of maternal corticosteroid on the fetal lung has been shown to take place at steroid levels comparable to those reached during the postnatal surge, with little or no additional response to higher doses (6).

The effects of antenatally administered corticosteroids are known to vary considerably depending on the species, GA, and steroid dosage studied (12). Such factors might explain the difference between the present results and those of the only other study of the effect of antenatal exposure to a corticosteroid on lung hyaluronan, namely that by Ikegami et al. (27). They did not find any reduction in the hyaluronan concentration in the lungs of preterm lambs $(\mathrm{GA} 125 \mathrm{~d}$; term $=150 \mathrm{~d})$ exposed antenatally to one to four doses of corticosteroids and ventilated for $40 \mathrm{~min}$ after delivery. In that study (27), the postnatal ventilation and the shifts in lung fluid might also have influenced the lung hyaluronan concentration and lung water (28). In addition, Ikegami et al. (27) reported the lung hyaluronan concentrations in relation to the wet lung weight, but not to the dry lung weight. In the present study, the results concerning the lung hyaluronan concentration are presented in relation to both dry and wet lung weights. The significant differences found between groups of pups concerning lung hyaluronan/dry lung weight were also observed when the hyaluronan concentration was related to the wet lung weight.

The lung water content did not differ between rabbit pups exposed to one antenatal dose of betamethasone and control pups in the present study. Only in pups delivered at GA $25 \mathrm{~d}$ and exposed to two doses of betamethasone, which led to a reduction of the lung hyaluronan concentration compared with that in the controls, was the lung water content higher than in control pups of the same GA. This finding concerning lung water is in agreement with two other reports. In preterm rabbit pups delivered at GA 28 d, von Stepinska et al. (29) found an increase in the pulmonary fluid content after the dams had received two doses of betamethasone on the days preceding delivery. The administered doses, each of $0.2 \mathrm{mg} / \mathrm{kg}$, were approximately equal to those given in the present study. In fetal rats, Schellenberg et al. (30) found an increase in lung water content after antenatal exposure to dexamethasone. In other studies the lung water content was found to be unaffected by antenatal steroid exposure, both in fetal rabbits $(14,31)$ and in preterm lambs (27). The lack of effect on the lung water content in most groups in the present study is also in accordance with our previous finding that changes in the hyaluronan concentration per dry lung weight are not paralleled by changes in the lung water content at birth (20).

The distribution of hyaluronan within the lung changes during fetal life (18), and as shown in this study, it can also be altered by antenatal administration of betamethasone. Hence, it is reasonable to speculate whether the lower hyaluronan concentration in the alveolar walls of pups delivered at GA 25 or $27 \mathrm{~d}$ and exposed to betamethasone antenatally, as observed in the lung sections stained for hyaluronan in the present study (Fig. 2), might contribute to a reduced amount of perialveolar lung water immediately after birth, and thus to improvement of gas diffusion between the alveoli and blood vessels.

Antenatally administered corticosteroids influence the lung concentration of different glycosaminoglycans in different ways. For example, the concentration of elastin has been found to be reduced, whereas that of collagen is unaffected, by administration of dexamethasone to fetal rats (30).

An additional finding in the present study was the absence of an effect of steroid exposure on the mean birth weight in pups delivered at GA 27 d. Retarded fetal growth after antenatal exposure of fetal rabbits to different doses of corticosteroids has been reported previously (13-15), but the effects described have varied. Rider et al. (14), for instance, found that in rabbit pups delivered at GA $27 \mathrm{~d}$, the highest betamethasone dose studied $(0.5 \mathrm{mg} / \mathrm{kg}$, given daily for $2 \mathrm{~d}$ before delivery) had the smallest effect on the birth weight. On the other hand, Sun et al. (15) found that the birth weights of rabbit pups delivered at GA $27 \mathrm{~d}$ were lower after exposure to a higher than to a lower dose of corticosteroids. These authors also noted a dissociation between growth retardation and lung maturation.

Antenatal corticosteroid exposure lowers the lung hyaluronan concentration at birth in preterm rabbit pups delivered at 
GA 25 or $27 \mathrm{~d}$, but not in those delivered at GA 28 or $29 \mathrm{~d}$. This lower lung hyaluronan concentration is accompanied by clearly less intense staining for hyaluronan in the subepithelial tissue in the alveolar walls. The lung water content at birth is not altered by the administration of one dose of betamethasone in pups delivered at any of the GAs studied. Administration of two equal doses of betamethasone preceding delivery at GA $25 \mathrm{~d}$ does not result in a lower lung hyaluronan concentration at birth than that seen in pups exposed to only one dose of betamethasone. However, in pups exposed to two doses of betamethasone, the lung water content at birth is higher than that in controls.

Acknowledgment. The authors thank Barbro Kjällström for expert technical assistance.

\section{REFERENCES}

1. Liggins GC, Howie RN 1972 A controlled trial of antepartum glucocorticoid treatment for prevention of the respiratory distress syndrome in premature infants. Pediatrics 50:515-525

2. NIH Consensus Development Panel 1995 Effect of corticosteroids for fetal maturation on perinatal outcome. JAMA 273:413-418

3. Crowley P, Chalmers I, Keirse MJNC 1990 The effect of corticosteroid administration before preterm delivery: an overview of the evidence from controlled trials. $\mathrm{Br} J$ Obstet Gynaecol 97:11-25

4. Ballard PL, Ballard RA 1995 Scientific basis and therapeutic regimens for use of antenatal glucocorticoids. Am J Obstet Gynecol 173:254-262

5. Jobe AH, Mitchell BR, Gunkel JH 1993 Beneficial effects of the combined use of prenatal corticosteroids and postnatal surfactant on preterm infants. Am J Obstet Gynecol 168:508-513

6. Gross I, Ballard PL 1998 Hormonal therapy for prevention of respiratory distress syndrome. In: Polin RA, Fox WW (eds) Fetal and Neonatal Physiology, 2nd Ed. WB Saunders, Philadelphia, pp 1314-1321

7. Kikkawa Y, Kaibara M, Motoyama EK, Orzalesi MM, Cook CD 1971 Morphological development of fetal rabbit lung and its acceleration with cortisol. Am J Pathol 64:423-433

8. Liley HG, White RT, Benson BJ, Ballard PL 1988 Glucocorticoids both stimulate and inhibit production of pulmonary surfactant protein A in fetal human lung. Proc Natl Acad Sci USA 85:9096-9100

9. Frank L 1992 Prenatal dexamethasone treatment improves survival of newborn rats during prolonged high $\mathrm{O}_{2}$ exposure. Pediatr Res 32:215-221

10. Warburton D, Parton L, Buckley S, Cosico L, Enns G, Saluna T 1988 Combined effects of corticosteroid, thyroid hormones, and $\beta$-agonist on surfactant, pulmonary mechanics, and $\beta$-receptor binding in fetal lamb lung. Pediatr Res 24:166-170
11. Liggins GC 1969 Premature delivery of foetal lambs infused with glucocorticoids. J Endocrinol 45:515-523

12. Seidner S, Pettenazzo A, Ikegami M, Jobe A 1988 Corticosteroid potentiation of surfactant dose response in preterm rabbits. J Appl Physiol 64:2366-2371

13. Ikegami M, Jobe AH, Seidner S, Yamada T 1989 Gestational effects of corticosteroids and surfactant in ventilated rabbits. Pediatr Res 25:32-37

14. Rider ED, Jobe AH, Ikegami M, Yamada T, Seidner S 1990 Antenatal betamethasone dose effects in preterm rabbits studied at 27 days gestation. J Appl Physiol 68:1134114

15. Sun B, Jobe A, Rider E, Ikegami M 1993 Single dose versus two doses of betamethasone for lung maturation in preterm rabbits. Pediatr Res 33: 256-260.

16. Franzblau C, Hayes JA, Snider GL 1977 Biochemical insights into the development of connective tissue I-II. In: Hodson WA (ed) Development of the Lung. Marcel Dekker, New York, pp 367-373

17. Fraser JRE, Laurent TC 1996 Hyaluronan. In: Comper WD (ed) Extracellular Matrix, Vol 2. Harwood Academic Publishers GmbH, Amsterdam, pp 141-199

18. Allen SJ, Sedin G, Jonzon A, Wells AF, Laurent TC 1991 Lung hyaluronan during development: a quantitative and morphological study. Am J Physiol 260:H1449H1454

19. Juul SE, Kinsella MG, Jackson JC, Truog WE, Standaert TA, Hodson WA 1994 Changes in hyaluronan deposition during early respiratory distress syndrome in premature monkeys. Pediatr Res 35:238-243

20. Johnsson H, Eriksson L, Jonzon A, Laurent TC, Sedin G 1998 Lung hyaluronan and water content in preterm and term rabbit pups exposed to oxygen or air. Pediatr Res 44:716-722

21. Barrada MI, Blomquist CH, Kotts C 1980 The effects of betamethasone on fetal development in the rabbit. Am J Obstet Gynecol 136:234-238

22. Johnsson H, Sedin G 1997 Antenatal corticosteroids lower the hyaluronan concentration in the lungs of preterm rabbit pups. Pediatr Res 42:413A(abstr)

23. Johnsson H, Eriksson L, Sedin G 1998 Antenatal corticosteroids lower the hyaluronan concentration in the lungs of preterm rabbit pups. Early Hum Dev 51:254(abstr)

24. Hellström S, Tengblad A, Johansson C, Hedlund U, Axelsson E 1990 An improved technique for hyaluronan histochemistry using microwave irradiation. Histochem J 22:677-682

25. Ripellino JA, Klinger MM, Margolis RU, Margolis RK 1985 The hyaluronic acid binding region as a specific probe for the localization of hyaluronic acid in tissue sections. J Histochem Cytochem 33:1060-1066

26. Brandt R, Hedlöf E, Asman I, Bucht A, Tengblad A 1987 A convenient radiometric assay for hyaluronan. Acta Otolaryngol Suppl (Stockh) 442:31-35

27. Ikegami M, Jobe AH, Newnham J, Polk DH, Willet KE, Sly P 1997 Repetitive prenatal glucocorticoids improve lung function and decrease growth in preterm lambs. Am J Crit Care Med 156:178-184

28. Lebel L, Smith L, Risberg B, Gerdin B, Laurent TC 1988 Effect of increased hydrostatic pressure on lymphatic elimination of hyaluronan from sheep lung. J Appl Physiol 64:1327-1332

29. von Stepinska J, Zbislawska P, Skoczowska W, Cretti A 1989 Der Vergleich des Einflusses von Lasolvan und von Betamethason auf die Lungenreife fetaler Kaninchen. Zentalbl Gynakol 111:379-384

30. Schellenberg JC, Liggins GC, Stewart AW 1987 Growth, elastin concentration, and collagen concentration of perinatal rat lung: effects of dexamethasone. Pediatr Res 21:603-607

31. Kotas RV, Mims LC, Hart LK 1974 Reversible inhibition of lung cell number after glucocorticoid injection into fetal rabbits to enhance surfactant appearance. Pediatrics $53: 358-361$ 